\title{
Antioxidant vitamins increase the collagen content and reduce MMP-1 in a porcine model of atherosclerosis: implications for plaque stabilization
}

\author{
J. Orbe, J.A. Rodríguez, R. Arias, M. Belzunce, B. Nespereira, M. Pérez-Ilzarbe, \\ C. Roncal, J.A. Páramo* \\ Atherosclerosis Research Laboratory, Division of Cardiovascular Pathophysiology, School of Medicine, University of Navarra, C/Irunlarrea 1, CIFA, E- \\ 31008 Pamplona, Spain
}

Received 23 July 2002; received in revised form 18 October 2002; accepted 25 October 2002

\begin{abstract}
Degradation of extracellular matrix, particularly interstitial collagen, promotes plaque instability and contributes to restenosis after vascular injury. We have explored the effects of vitamins C and E on the collagen content and metalloproteinase-1 (MMP-1) expression after angioplasty in hypercholesterolemic pigs. Iliac angioplasty was performed on 18 minipigs divided into three diet groups: a normal-cholesterol (NC), a high-cholesterol (HC) and a high-cholesterol plus vitamins $\mathrm{C}+\mathrm{E}(\mathrm{HCV})$. Four weeks later, after sacrifice, the vascular collagen content and MMP-1 protein expression, along with the plasma caseinolytic activity and lipid peroxidation, were measured. MMP-1 was also determined in arterial rings stimulated with native low-density lipoproteins (LDL) isolated from experimental groups. Cholesterol-rich diet augmented plasma lipid peroxidation $(P<0.05)$, reduced the collagen content and increased vascular MMP-1 expression after injury $(P<0.05)$. Enhanced caseinolytic activity (identified as MMP-1) was also observed in HC plasma samples and in supernatants from arterial rings incubated with HC-LDL. Vitamins C and E markedly increased neointimal collagen content $(P<0.01)$, reduced the hypercholesterolemia-induced changes in vascular MMP-1 $(P<0.05)$ and diminished plasma and ex vivo caseinolytic activity. Vitamins $C$ and $E$ may help stabilize atherosclerotic plaque after angioplasty and favor vascular remodeling by increasing collagen content and reducing vascular MMP-1 expression in porcine hypercholesterolemia.
\end{abstract}

(C) 2002 Elsevier Science Ireland Ltd. All rights reserved.

Keywords: Antioxidant vitamins; Hypercholesterolemia; Atherosclerosis; Collagen; Metalloproteinase-1

\section{Introduction}

Degradation of extracellular matrix (ECM) contributes crucially to a variety of processes, including atherosclerosis and restenosis after vascular injury and is a critical event leading to the weakening of the vessel wall $[1,2]$. ECM degradation by metalloproteinases (MMPs) is known to be involved in the pathogenesis of cardiovascular diseases, including atherosclerosis, restenosis and myocardial infarction [3]. MMPs are a family of zinc-dependent enzymes that can be divided

\footnotetext{
* Corresponding author. Tel.: +34-948-425-600; fax: +34-948-425652 .

E-mail address: japaramo@unav.es (J.A. Páramo).
}

into two structurally distinct groups, such as secreted MMPs and membrane-type MMPs. Secreted MMPs include collagenases (MMP-1, MMP-8), gelatinases (MMP-2, MMP-9) stromelysins (MMP-3, MMP-10) and other MMPs. MMP-1 secreted from cells present in atheroma could be involved in the subendothelial destruction of ECM and remodeling of plaques [3,4].

Previous studies have demonstrated that hypercholesterolemia induces local collagen loss, contributing to plaque destabilization [5]. Although the mechanisms involved in these processes are still unclear, it has been suggested that the activation of cells in atheroma could be a principle source of MMPs. oxLDL stimulate macrophage activation and induce the expression of MMP-1 by vascular cells [6]; this proteinase is known to play a prominent role in plaque stability [7] and, in 
addition, lipid lowering has been shown to reduce MMP-1 activity and increase collagen content of atheroma [8].

Antioxidant vitamins have been evaluated through clinical studies for their association with atherosclerotic disease, but their results are not conclusive. Recent clinical trials enrolling patients with established atherosclerosis or at high vascular risk, have failed to demonstrate that vitamin $\mathrm{E}$ reduces the progression of the disease [9]. In contrast, other studies have demonstrated that antioxidant vitamins retard the development of coronary arteriosclerosis [10]. In experimental models, we and others have previously shown that antioxidants are able to reduce intimal lesions, decrease type-1 plasminogen activator inhibitor (PAI-1) after injury and restore endothelial function [11-13], but their possible effect on the regulation of MMPs and/or neointimal collagen content has not been properly assessed.

The pig model appears to be the most relevant to human restenosis because of its similarities with human coronary circulation, spontaneous development of atherosclerosis and histological response to vascular injury [14]. We hypothesized that antioxidant vitamins $\mathrm{C}$ and $\mathrm{E}$ would preserve the collagen content in a hypercholesterolemic porcine model of angioplasty, possibly by reducing the MMP-1 expression. Treatment with vitamins was initiated 3 weeks after the induction of hypercholesterolemia in order to more closely mimic a potential clinical situation.

\section{Material and methods}

\subsection{Animals}

Eighteen Yucatan miniature pigs (4 months old, mean weight $33.29 \pm 1.69 \mathrm{~kg}$ ) were obtained from our breeding center and maintained in the animal facilities at CIFA (Centro de Investigación en Fármaco-Biología Aplicada), a GLP-accredited center within the University of Navarra (Pamplona, Spain). Housing conditions followed the European regulations (86/609/CEE).

\subsection{Biochemical measurements}

Concentrations of total serum cholesterol and its fractions were determined by standard enzymatic assays.

Plasma lipid peroxidation was measured as thiobarbituric acid reactive substances (TBARS) [15]. Vitamin $\mathrm{E}$ was measured in plasma using a reverse-phase highperformance liquid chromatography (HPLC) [13].

\subsection{Vascular injury and tissue processing}

Our model of vascular lesion after iliac angioplasty in hypercholesterolemic pigs has recently been described
[11]. Briefly, animals were divided into three groups $(n=6)$ and fed different dietary formulations for 4 weeks: a normal-cholesterol (NC) control group, given standard porcine chow (Porcisanders, Sanders); a highcholesterol (HC) group fed with a diet containing 24.5\% animal lard, 4\% cholesterol (Roig Farma) and 1.5\% biliar extract (Roig Farma); and a high-cholesterol plus vitamins $\mathrm{C}$ and $\mathrm{E}(\mathrm{HCV})$ group fed similar to the $\mathrm{HC}$ group, but supplemented during the last week with $1 \mathrm{~g}$ vitamin C and 1000 IU vitamin E (Roig Farma) per animal per day. The dosage of antioxidants was chosen from a similar model demonstrating that animals treated with vitamins $\mathrm{C}$ and $\mathrm{E}$ have a smaller degree of $\mathrm{LDL}$ oxidation and the best response to vascular injury [12]. At this time, the animals were subjected to catheterization in the right internal iliac artery, the left internal iliac artery was used as control. The animals from each group were allowed to recover and maintained their designated diets until they were sacrificed, 28 days after intervention.

Vascular tissues were fixed with paraformaldehyde $4 \%$, dehydrated and paraffin-embedded for histological purposes. Arterial blood samples were drawn before and after arterial injury for analytical measurements.

\subsection{Immunohistological analysis}

Sirius red staining was used to identify interstitial collagen on serial $3 \mu \mathrm{m}$ sections as previously described [16].

Tissue sections for MMP-1 immunohistochemistry were heated in sodium citrate buffer $(0.01 \mathrm{M}, \mathrm{pH}=6)$ for antigen retrieval before their incubation with $2 \mu \mathrm{g} / \mathrm{ml}$ murine anti-MMP-1, which recognizes both active and latent forms (IM35L Oncogen, USA), $0.5 \mu \mathrm{g} / \mathrm{ml}$ murine anti- $\alpha$-smooth muscle actin (M850, Dako, Denmark) and murine anti-swine leukocyte Ag monoclonal antibody 4E9 [17] (kindly provided by Dr J. Dominguez, INIA, Spain). All sections were incubated with secondary biotinylated rabbit anti-mouse Fc (Dako), developed with avidin-biotin-peroxidase complex (ABC, Dako, Denmark) and 3-3'-diaminobenzidine hydrochloride (Sigma, USA) and counterstained with Harris' hematoxylin [11].

A visual grading scale was used for assessing the intensity of MMP-1 immunostaining. Three observers, independently and in a blinded fashion, characterized sections by comparing them against a control section using the grading scale. Each observer evaluated and scored each field on a 0 to $3+$ scale for the extent distribution of positive staining [11].

\subsection{Effect of porcine $L D L$ on $M M P-1$ ex vivo expression}

Coronary artery rings $(n=9)$ were incubated for $48 \mathrm{~h}$ with native LDL $(100 \mu \mathrm{g}$ protein $/ \mathrm{ml})$ isolated from 


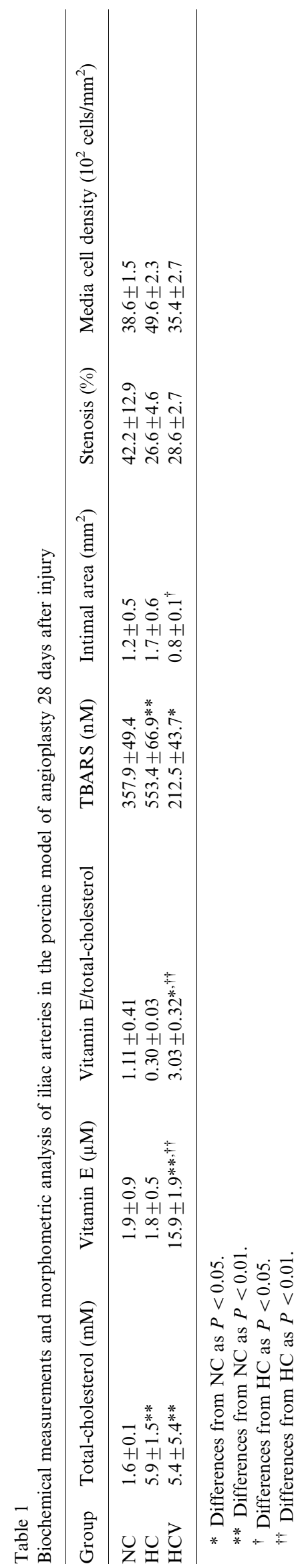

porcine plasma from experimental groups $(\mathrm{NC}, \mathrm{HC}$ and HCV) by density gradient ultracentrifugation [18]. Supernatants of cultured rings were separated by SDSPAGE under reducing conditions in Tris-glycine-SDS buffer ( $\mathrm{pH} 8.3$ ), electroblotted onto nitrocellulose membrane and probed with murine anti-MMP-1 $(1 \mu \mathrm{g} / \mathrm{ml})$, followed by a peroxidase-linked rabbit anti-mouse $\mathrm{IgG}$ (1:2000, Zymed, USA). Blots were developed using a chemiluminescence system (ECL, AmershamBioscience), on X-ray film. Human recombinant MMP-1 (Amersham Bioscience) was used as the positive control.

\subsection{Zymographic analysis}

Some $10 \mu \mathrm{l}$ of coronary supernatants or a 1:20 dilution of plasma samples in saline were loaded on a standard $8 \%$ SDS-PAGE gel containing $0.5 \%$ casein and electrophoresed under non-reducing conditions. Substrate gels were soaked with Triton X-100 solution $(2.5 \%)$ for $15 \mathrm{~min}$ at room temperature and then incubated overnight at $37{ }^{\circ} \mathrm{C}$ with a $50 \mathrm{mM}$ Tris- $\mathrm{HCl}$ buffer $\left(\mathrm{pH} 7.4,0.05 \% \mathrm{NaCl}, 0.1 \% \mathrm{NaN}_{3}\right.$ and $10 \mathrm{mM}$ $\mathrm{CaCl}_{2}$ ). The lysis of the casein in the gel was visualized by staining with Coomassie brilliant blue (Sigma), densitometrically quantified on a GS690 system (Bio$\mathrm{Rad}$ ) and identified on the basis of protein electrophoretic mobility [19]. Quenching analysis were performed by simultaneous incubation of plasma samples with 5-50 ng of a monoclonal antibody anti-MMP-1 (Oncogene).

\subsection{Statistical analysis}

All data was expressed as mean \pm S.E.M. Statistically significant differences between groups were calculated by analysis of variance (ANOVA) followed by Tukey posthoc test or Kruskal-Wallis and Mann-Whitney test, for parametric and non-parametric conditions, respectively. Statistical significance was accepted at the 95\% confidence level $(P<0.05)$. Association between variables was assessed by using the Spearman's correlation test. Analysis was performed by using the computer program SPSS for Windows (SPSS Inc).

\section{Results}

\subsection{Plasma cholesterol, lipid peroxidation and lesion formation}

As shown in Table 1, pigs receiving hypercholesterolemic diet showed higher cholesterol concentration $(P<0.01)$ and plasma lipid peroxidation $(P<0.01)$ than the controls. The angioplasty induced severe structural changes in the vascular wall in comparison 


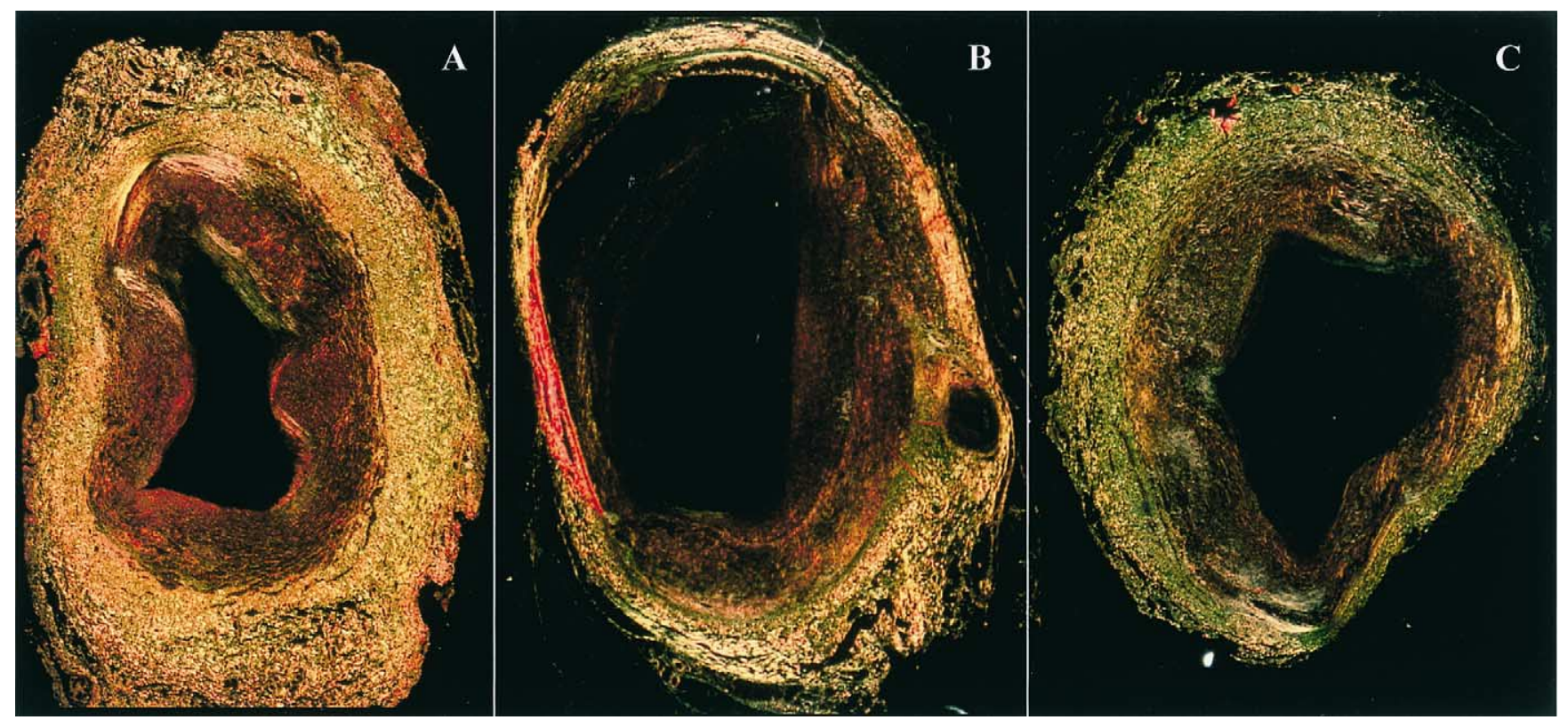

Fig. 1. Sirius red staining of vascular tissue sections from the three experimental groups, photographed with a polarizing filter. Intense collagen loss was observed in the injured $\mathrm{HC}$ arteries (panel B) in comparison with the NC group (panel A). Treatment with vitamins $\mathrm{C}$ and $\mathrm{E}$ induced a significant increase in the collagen content of vascular sections (panel C).

with uninjured arteries, resulting in an increased intimal area and media cell density in $\mathrm{HC}$ group compared to the NC group (data not shown).

Treatment with vitamins $\mathrm{C}$ and $\mathrm{E}$ had no effect on total cholesterol or LDL concentration, but, as expected, it increased plasma vitamin E levels and, particularly, the vitamin $\mathrm{E}$ content in the LDL fraction compared with $\mathrm{HC}(14.5 \pm 3.6$ vs. $4.29 \pm 2.1 \mathrm{nM} / \mathrm{mg}$ protein, $P<0.05)$. As shown in Table 1 , antioxidant treatment significantly reduced the hypercholesterolemia-induced plasma lipid peroxidation at the time of sacrifice $(P<0.01)$, without significant improvement in morphologic parameters, except a slight, but significant, reduction in the intimal area $(P<0.05)$.

\subsection{Atherosclerotic lesion collagen content}

Vascular sections stained for collagen with Sirius red were analyzed under polarized light. As shown in Fig. 1, the lesions in the NC group were intensely stained for collagen fibers forming intricate networks throughout the plaque (Fig. 1A). However, the collagen content was practically absent throughout the vascular wall in the HC group (Fig. 1B), whereas a significant recovery was observed in the HCV group (Fig. 1C). The collagen stained area in plaques was 2.4-fold higher in the $\mathrm{NC}$ group than in $\mathrm{HC}$ group $(70.8 \pm 8.1 \%$ vs. $29.3 \pm 8.9 \%$, $P<0.01)$. Antioxidant vitamins $\mathrm{C}$ and $\mathrm{E}$ significantly restored the collagen content with respect to $\mathrm{HC}(65.5 \pm$ $7.4 \%$ vs. $29.3 \pm 8.9 \%, P<0.01)$ without differences regarding the $\mathrm{NC}$ group.

\subsection{MMP activity}

Since no vascular samples were available for zymography, we performed caseinolytic analysis of plasma samples in order to determine whether hypercholesterolemia induces increased systemic proteolytic activity. A similar proteolytic profile activity was observed in all of the experimental groups, but a more intense $52 \mathrm{kDa}$ proteolytic band was found in the samples obtained after injury in the HC group than in the NC group $(P<$ 0.05, Fig. 2A). However, as shown in Fig. 2(A), the 52 $\mathrm{kDa}$ caseinolytic activity was reduced in the group treated with antioxidants in comparison to the $\mathrm{HC}$ group, indicating that in addition to local effects on collagen content, vitamins $\mathrm{C}$ and $\mathrm{E}$ also reduced the systemic proteolytic activity. In order to identify the caseinolytic band, additional experiments were performed by preincubating plasma samples with different concentrations of specific MMP-1 antibody. As shown in Fig. 2(B), a dose-dependent reduction in the proteolytic activity was found, indicating that it corresponds to MMP-1.

Taking a further step, we performed immunohistochemical analysis for MMP-1 in vascular sections in 
$\mathbf{A}$

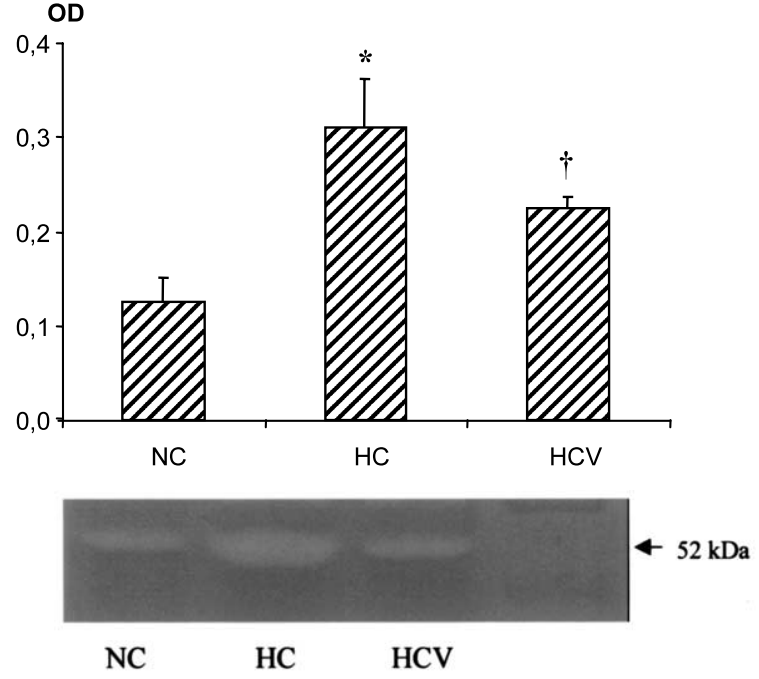

B

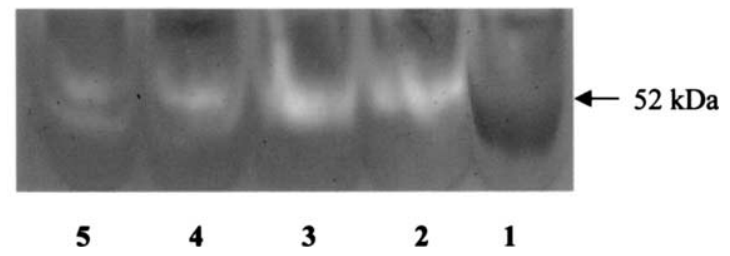

Fig. 2. Caseinolytic activity of plasma samples and quantitative analysis (top) of proteolytic activity in $\mathrm{NC}, \mathrm{HC}$ and $\mathrm{HCV}$ groups (A). Quenching experiments performed by simultaneous incubation of plasma samples with different concentrations $(5-50 \mathrm{ng})$ of a monoclonal antibody anti-MMP-1 show the specificity of the caseinolytic activity (B). Lane 1: molecular weight marker; lane 2: HC plasma sample; lane 3-5: plasma sample preincubated with 5, 25 and $50 \mathrm{ng}$ of murine anti-MMP-1 antibody respectively. ${ }^{*} P<0.05$ in comparison with the NC group. $\uparrow P<0.05$ in comparison with $\mathrm{HC}$ group.

order to assess whether MMP-1 expression could be associated with observed modifications in collagen content within vascular vessels in the experimental groups. As shown in Fig. 3, MMP-1 protein was significantly increased in the neointima and media layers of $\mathrm{HC}$ group compared to $\mathrm{NC}(P<0.05$, Fig. 3A, B). Median quantitative score for MMP-1 immunostaining was also found to be significantly higher $(P<0.05)$ in the HC group (Table 2). The most intense signal was observed within the vascular lesion, near the media and in the thickened intima (Fig. 3D). Areas with the most intense signal were also positive for $\alpha$-actin smooth muscle cells (SMC) and macrophages, suggesting that these cellular types are involved in MMP-1 vascular expression (Fig. 3E, F).

Interestingly, the antioxidant treatment was able to significantly reduce the observed vascular MMP-1 expression in response to injury in the $\mathrm{HCV}$ group. Immunohistochemical analysis showed weaker MMP-1 reactivity in the media and in the thickened intima in the group of pigs receiving antioxidants than in the $\mathrm{HC}$ group $(P<0.05)$ (Fig. 3C). Median quantitative score for MMP-1 immunostaining was found to be significantly lower $(P<0.05)$ in the HCV group than in the HC group (Table 2).

\subsection{Effect of native LDL on MMP-1 activity and expression}

Ex vivo experiments performed with supernatants from cultured porcine coronary rings showed an increased $52 \mathrm{kDa}$ caseinolytic activity in the segments incubated with LDL obtained from the HC group (Fig. 4A). A weaker ex vivo proteolytic activity could be observed in the conditioned medium from coronary rings treated with HCV-LDL than those treated with HC-LDL; the said activity was later identified as MMP1 by Western blot analysis (Fig. 4B).

\section{Discussion}

Degradation of ECM is a critical event leading to the weakening of the vessel wall. A mechanistic link between hypercholesterolemia and collagen loss is still hypothetical, although a critical role of MMPs and other proteolytic enzymes have also been strongly suggested [5]. In the present study, we report that a combined dietary treatment of vitamins $\mathrm{C}$ and $\mathrm{E}$ restores the hypercholesterolemia-induced collagen loss and reduces both vascular and systemic MMP-1 after vascular injury in pigs. This effect was largely independent of lipid lowering and occurred despite trivial morphologic changes in the vessel wall.

We had previously shown that hypercholesterolemia induces severe structural and morphologic changes in the vessel wall after angioplasty [11]. This study extends those findings by showing that Sirius red stained injured arteries from hypercholesterolemic pigs exhibit extensive areas devoid of collagen filaments (coinciding with recent reports [20]) suggesting an increased proteolytic activity or diminished collagen synthesis. The zymographic analysis of plasma samples displaying an intense MMP-1 caseinolytic band as well as an increase in vascular MMP-1 protein expression would support those findings. Areas positively stained for MMP-1 were observed mainly in the media and in the intimal thickening close to internal elastic lamina in the $\mathrm{HC}$ group, thereby confirming previous reports [21-23]. MMP-1 has been shown to be specifically present in atherosclerotic lesions [6,24], secreted by endothelial cells, SMC and macrophages and could be involved in the ECM degradation, which is considered an important feature for plaque stability $[7,25]$. Our results show that areas with the most intense signal were also positive for SMC and macrophages, confirming that both cellular types are involved in the MMP-1 vascular expression. 


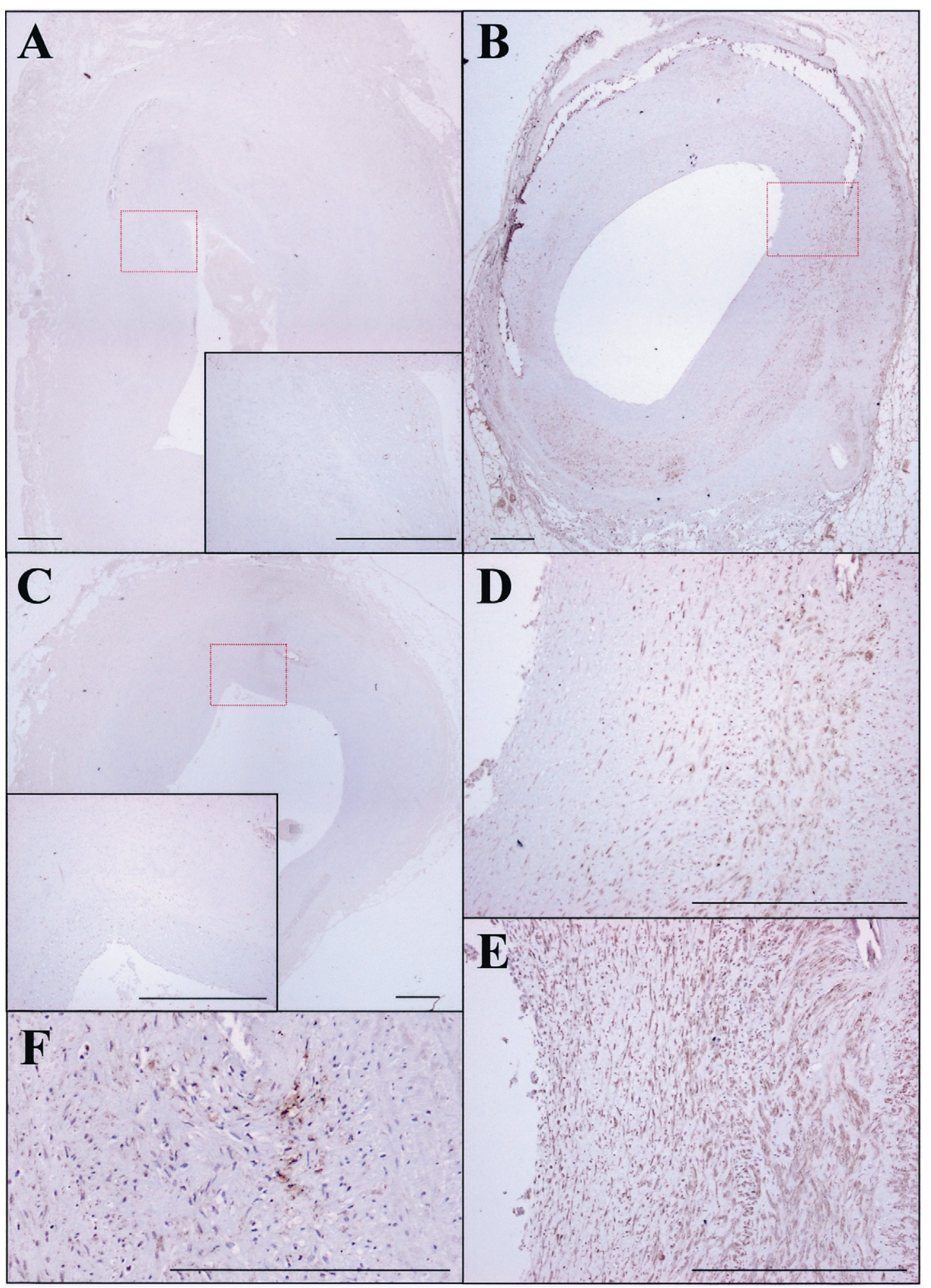

Fig. 3. Immunohistochemical pattern for vascular MMP-1 in the different groups of pigs. Whereas a weak MMP-1 signal (score $=1$ ) was present in $\mathrm{NC}$ (panel A) in comparison with the HC (score = 3) (B), the vascular immunoreactive MMP-1 expression after angioplasty was significantly reduced $($ score $=1)$ in hypercholesterolemic pigs treated with vitamins $\mathrm{C}$ and $\mathrm{E}$ (panel $\mathrm{C}$ ), thereby being similar to NC. Areas squared in red correspond to magnified details. Panel D shows a magnified detail of (B). Panels E and F show the smooth muscle $\alpha$-actin (E) and macrophages (F) staining of the same region. Scale bar, $400 \mu \mathrm{m}$. 


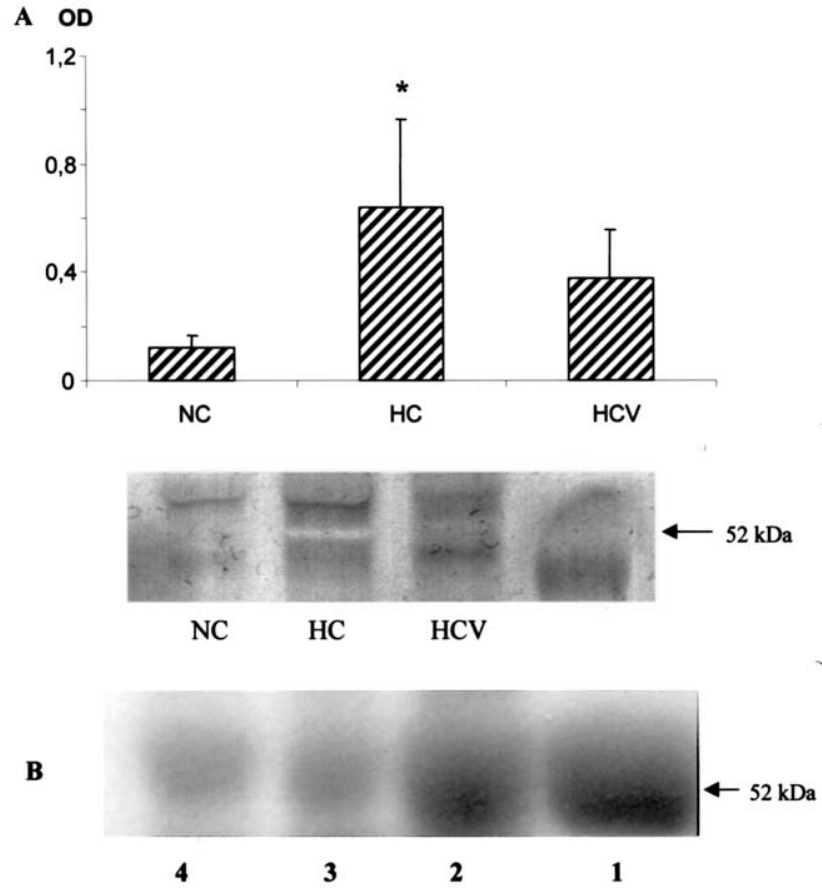

Fig. 4. Effect of purified LDL on MMP-1 induced in coronary artery rings. Caseinolytic activity of supernatants from LDL-stimulated porcine rings and quantitative analysis (top) of caseinolytic activity in $\mathrm{NC}, \mathrm{HC}$ and $\mathrm{HCV}$ groups (A). Western blotting for MMP-1 in supernatants of cultured aortic rings (B). Lane 1: standard of human recombinant MMP-1 (52 kDa). Supernatants from cultures incubated with LDL from HC (lane 2), HCV (lane 3) and NC (lane 4), all three showing a positive band at $52 \mathrm{kDa}$ size.

Table 2

Semiquantitative score assessment of vascular MMP-1 in the porcine iliac artery by immunohistochemistry

\begin{tabular}{lllllll}
\hline \multirow{2}{*}{ Group } & Group size & \multicolumn{3}{l}{ Score } & \multicolumn{3}{c}{ Median score } \\
\cline { 3 - 5 } & & 0 & 1 & 2 & 3 & \\
\hline NC & 6 & 2 & 3 & 1 & 0 & 1 \\
HC & 6 & 0 & 0 & 3 & 3 & $2.5^{*}$ \\
HCV & 6 & 0 & 4 & 2 & 0 & 1 \\
\hline
\end{tabular}

* $P<0.05$ in comparison with both NC and HCV.

In a further step, we performed ex vivo experiments in cultured normal coronary rings to assess the effect of native LDL, from the three experimental groups, on MMP-1. The fact that MMP-1 was increased after stimulation with LDL from hypercholesterolemic pigs would support the idea that hypercholesterolemia plays a direct role in the induction of MMP-1 by vascular cells. According to this data, hypercholesterolemia would play a significant role in the secretion of MMP1 by cells present within the atheroma.

For the first time, we provide evidence that antioxidant vitamins $\mathrm{C}$ and $\mathrm{E}$, despite a minimum effect on morphologic changes in the vascular wall, can signifi- cantly increase the collagen content in hypercholesteromic injured arteries, which could be related to a significant reduction of vascular MMP-1. That effect was independent of lipid lowering because vitamin treatment did not alter plasma cholesterol levels. Moreover, the systemic proteolytic activity was also attenuated in the plasma of the pigs receiving antioxidants. Finally, ex vivo incubation of porcine coronary arteries with native LDL from hypercholesterolemic pigs treated with vitamins $\mathrm{C}$ and $\mathrm{E}$ attenuated the enhancement of caseinolytic activity induced by hypercholesterolemia. Based on aforementioned data together, it appears that the combination of vitamins $\mathrm{C}$ and $\mathrm{E}$ could reduce the hypercholesterolemia-induced MMP-1 expression in our experimental model of vascular injury and, therefore, prevent ECM degradation by increasing the collagen content, thus contributing to plaque stabilization. Other studies have reported a slow progression of atherosclerosis in experimental models using antioxidant vitamins $C$ and $E[11,12,26]$. In addition, it has been recently shown that vitamin $\mathrm{C}$ deficiency induces a type of plaque morphology that is potentially vulnerable to rupture [27].

The mechanism by which antioxidants may participate in matrix-protective processes remains unclear. We show herein that the combination of vitamins $\mathrm{C}+\mathrm{E}$ reduces plasma lipid peroxidation. Oxidative stress is increasingly being recognized as a potentially important contributor to atherogenesis and restenosis after vascular interventions and injury [28,29]. The generation of reactive oxygen species and oxidation of lipids have profound and wide-range effects that can dramatically increase vascular toxicity and initiate a cascade of molecular and cellular responses. Increased vascular expression of MMP-1 in hypercholesterolemia can be a direct effect of oxidative stress because reactive oxygen species are known to enhance MMP-1 expression and activity in vitro [30,31]. Our results, which show that dietary supplementation with vitamins $\mathrm{C}+\mathrm{E}$ was able to significantly reduce plasma lipid peroxidation, support this hypothesis. In fact, constituents of oxidatively modified lipoproteins that stimulate protein kinase $\mathrm{C}$ (PKC) might link to the activator protein-1 (AP-1)mediated transcriptional regulation that plays a key role in controlling transcription of MMP-1 [8]. Moreover, $\mathrm{NF}-\kappa \mathrm{B}$, a transcription factor activated by oxidative stress/free radicals [32], has been shown to be required for cytokine upregulation of MMP-1 in vascular cells [33]. $\alpha$-Tocopherol is a PKC inhibitor capable of diminishing MMP-1 expression in fibroblast cultures [34], but it can also inhibit $\mathrm{NFkB}$, resulting in a significant inhibition of monocyte cell adhesion or cytokine regulation, which is required for upregulation of MMP-1 in in vitro studies [33,35].

Our study has some limitations. Other MMPs are also playing a role in arterial remodeling after injury and 
ECM degradation. In immunohistochemical staining, the presence of MMPs assessed by immunohistochemistry does not imply increased catalytic capacity [23]. In addition, matrix catabolism in the cardiovascular system involves enzymes other than the MMP family, such as cathepsins and cystatins.

In conclusion, the present study is the first to show an increase in the neointimal collagen content and a reduction of vessel-wall MMP-1 in a porcine hypercholesterolemic model of vascular injury in response to vitamins $\mathrm{C}$ and $\mathrm{E}$, thereby suggesting that antioxidant therapy can contribute to prevent matrix degradation in atherosclerosis, thus contributing to plaque stabilization.

\section{Acknowledgements}

This study was supported by grants from the Departamentos de Salud y Educación (Gobierno de Navarra) and PIUNA from the University of Navarra and Grant 97/0940 from FIS (Ministerio de Sanidad, Spain).

\section{References}

[1] Kakuta T, Usui M, Coats WD, Currier JW, Numano F, Faxon DP. Arterial remodeling at the reference site after angioplasty in the atherosclerotic rabbit model. Arterioscler Thromb Vasc Biol 1998; 18:47-51.

[2] de Smet BJ, Pasterkamp G, van der Helm YJ, Borst C, Post MJ. The relation between de novo atherosclerosis remodeling and angioplasty-induced remodeling in an atherosclerotic Yucatan micropig model. Arterioscler Thromb Vasc Biol 1998;18:702-7.

[3] Lee RT, Schoen FJ, Loree HM, Lark MW, Libby P. Circumferential stress and matrix metalloproteinase 1 in human coronary atherosclerosis. Implications for plaque rupture. Arterioscler Thromb Vasc Biol 1996;16:1070-3.

[4] Shapiro SD. Matrix metalloproteinase degradation of extracellular matrix: biological consequences. Curr Opin Cell Biol 1998; 10:602-8.

[5] Rekhter MD, Hicks GW, Brammer DW, et al. Hypercholesterolemia causes mechanical weakening of rabbit atheroma: local collagen loss as a prerequisite of plaque rupture. Circ Res 2000;86:101-8.

[6] Huang Y, Mironova M, Lopes Virella MF. Oxidized LDL stimulates matrix metalloproteinase-1 expression in human vascular endothelial cells. Arterioscler Thromb Vasc Biol 1999;19:2640-7.

[7] Sukhova GK, Schonbeck U, Rabkin E, Schoen FJ, Poole AR, Billinghurst RC, Libby P. Evidence for increased collagenolysis by interstitial collagenases- 1 and -3 in vulnerable human atheromatous plaques. Circulation 1999;99:2503-9.

[8] Aikawa M, Rabkin E, Okada Y, et al. Lipid lowering by diet reduces matrix metalloproteinase activity and increases collagen content of rabbit atheroma: a potential mechanism of lesion stabilization. Circulation 1998;97:2433-44.

[9] MRC/BHF. Heart protection study of antioxidant vitamin supplementation in 20,536 high-risk individuals: a randomised placebo-controlled trial. Lancet 2002;360:23-33.
[10] Fang JC, Kinlay S, Beltrame J, et al. Effect of vitamins C and E on progression of transplant-associated arteriosclerosis: a randomised trial. Lancet 2002;359:1108-13.

[11] Orbe J, Rodriguez JA, Calvo A, et al. Vitamins C and E attenuate plasminogen activator inhibitor-1 (PAI-1) expression in a hypercholesterolemic porcine model of angioplasty. Cardiovasc Res 2001;49:484-92.

[12] Nunes GL, Sgoutas DS, Redden RA, Sigman SR, Gravanis MB, King SB, III, Berk BC. Combination of vitamins $\mathrm{C}$ and $\mathrm{E}$ alters the response to coronary balloon injury in the pig. Arterioscler Thromb Vasc Biol 1995;15:156-65.

[13] Rodriguez JA, Grau A, Eguinoa E, et al. Dietary supplementation with vitamins $\mathrm{C}$ and $\mathrm{E}$ prevents downregulation of endothelial NOS expression in hypercholesterolemia in vivo and in vitro. Atherosclerosis 2002;165:33-40.

[14] de Smet BJ, van der Zande J, van der Helm YJ, Kuntz RE, Borst C, Post MJ. The atherosclerotic Yucatan animal model to study the arterial response after balloon angioplasty: the natural history of remodeling. Cardiovasc Res 1998;39:224-32.

[15] Conti M, Morand PC, Levillain P, Lemonnier A. Improved fluorometric determination of malonaldehyde. Clin Chem 1991;37:1273-5.

[16] Junqueira LC, Bignolas G, Brentani RR. Picrosirius staining plus polarization microscopy, a specific method for collagen detection in tissue sections. Histochem J 1979;11:447-55.

[17] Bullido R, Gomez del Moral M, Alonso F, et al. Monoclonal antibodies specific for porcine monocytes/macrophages: macrophage heterogeneity in the pig evidenced by the expression of surface antigens. Tissue Antigens 1997;49:403-13.

[18] Chapman MJ, Goldstein S, Lagrange D, Laplaud PM. A density gradient ultracentrifugal procedure for the isolation of the major lipoprotein classes from human serum. J Lipid Res 1981;22:33958.

[19] Fernandez Resa P, Mira E, Quesada AR. Enhanced detection of casein zymography of matrix metalloproteinases. Anal Biochem 1995;224:434-5.

[20] Theilmeier G, Quarck R, Verhamme P, et al. Hypercholesterolemia impairs vascular remodelling after porcine coronary angioplasty. Cardiovasc Res 2002;55:385-95.

[21] Zempo N, Kenagy RD, Au YP, Bendeck M, Clowes MM, Reidy MA, Clowes AW. Matrix metalloproteinases of vascular wall cells are increased in balloon-injured rat carotid artery. J Vasc Surg 1994;20:209-17.

[22] Bendeck MP, Irvin C, Reidy MA. Inhibition of matrix metalloproteinase activity inhibits smooth muscle cell migration but not neointimal thickening after arterial injury. Circ Res 1996;78:3843.

[23] Forough R, Koyama N, Hasenstab D, Lea H, Clowes M, Nikkari ST, Clowes AW. Overexpression of tissue inhibitor of matrix metalloproteinase-1 inhibits vascular smooth muscle cell functions in vitro and in vivo. Circ Res 1996;79:812-20.

[24] Hojo Y, Ikeda U, Takahashi M, et al. Matrix metalloproteinase-1 expression by interaction between monocytes and vascular endothelial cells. J Mol Cell Cardiol 2000;32:1459-68.

[25] Galis ZS, Sukhova GK, Lark MW, Libby P. Increased expression of matrix metalloproteinases and matrix degrading activity in vulnerable regions of human atherosclerotic plaques. J Clin Invest 1994:94:2493-503.

[26] Diaz MN, Frei B, Vita JA, Keaney JF. Antioxidants and atherosclerotic heart disease. New Engl J Med 1997;337:408-16.

[27] Nakata Y, Maeda N. Vulnerable atherosclerotic plaque morphology in apolipoprotein E-deficient mice unable to make ascorbic acid. Circulation 2002;105:1485-90.

[28] Huraux C, Makita T, Kurz S, et al. Superoxide production, risk factors, and endothelium-dependent relaxations in human internal mammary arteries. Circulation 1999;99:53-9. 
[29] Cai H, Harrison DG. Endothelial dysfunction in cardiovascular diseases: the role of oxidant stress. Circ Res 2000;87:840-4.

[30] Rajagopalan S, Meng XP, Ramasamy S, Harrison DG, Galis ZS Reactive oxygen species produced by macrophage-derived foam cells regulate the activity of vascular matrix metalloproteinases in vitro. Implications for atherosclerotic plaque stability. J Clin Invest 1996;98:2572-9.

[31] Siwik DA, Pagano PJ, Colucci WS. Oxidative stress regulates collagen synthesis and matrix metalloproteinase activity in cardiac fibroblasts. Am J Physiol Cell Physiol 2001;280:C53-60.

[32] Li N, Karin M. Is NF-kappaB the sensor of oxidative stress? FASEB J 1999;13:1137-43.
[33] Bond M, Chase AJ, Baker AH, Newby AC. Inhibition of transcription factor NF-kappaB reduces matrix metalloproteinase-1, -3 and -9 production by vascular smooth muscle cells. Cardiovasc Res 2001;50:556-65.

[34] Ricciarelli R, Maroni P, Ozer N, Zingg JM, Azzi A. Agedependent increase of collagenase expression can be reduced by alpha-tocopherol via protein kinase C inhibition. Free Rad Biol Med 1999;27:729-37.

[35] Chase AJ, Bond M, Crook MF, Newby AC. Role of nuclear factor-kappa B activation in metalloproteinase-1, -3, and -9 secretion by human macrophages in vitro and rabbit foam cells produced in vivo. Arterioscler Thromb Vasc Biol 2002;22:765-71. 